\title{
Methodology assistant in a graphical design of real-time applications
}

\author{
R. Aubry, M. Maranzana and J.J. Schwarz \\ LISPI - L3I - INSA de Lyon Département Informatique - Bât. 502 \\ F-69621 Villeurbanne Cedex, France \\ tel.: (+33) 72438162 \\ fax: (+33) 72438518 \\ e-mail: Mathieu.Maranzana@if.insa-lyon.fr
}

\begin{abstract}
LACATRE (Langage d'Aide à la Conception d'Applications Temps REel) is a graphical environment dedicated to multitasking real-time application developments. At the lowest level, it handles objects close to those supported by real-time executives (such as tasks, messages, semaphores...), and at the highest level, it manipulates applicative objects (like agencies and processes), near to the application programmer's preoccupation. Graphical programming has been used in order to obtain a documentation with a high degree of legibility and a synthetic as well as precise view of the dynamic behaviour of a real-time application.

The aim of this paper is to present the definition of a methodology assistant which takes advantage of the specificity of the Lacatre environment -behavioural axis, transformational axis, abstraction level axis, operative mode axis and graphical programming-, allowing an incremental development and providing an assistance adapted to the design context. Starting with a known, and rule based, stepwise design method, the methodology assistant can help the designer to verify the design steps and to monitor the complexity of the software components currently under construction.
\end{abstract}

\section{Keywords}

CASE tool, graphical design, real-time application, methodology, assistant, quality 


\section{INTRODUCTION}

If the major concern in the development of real-time applications is to produce functionally correct results within given deadlines, the same holds more and more true, with respect to the quality control, trying to reach in priority the maintainability and the reliability factors.

At the outset, the aim of the Lacatre tool was to provide our students with a set of pedagogical tools for real-time multitasking learning. But, in fact the use of such a tool is not only restricted to educational purposes: a lot of industrial applications are based on the use of multitasking real-time kernels. In such cases, the tool will bring a substantial aid in the creation of programmes and their documentation. The Lacatre environment relies upon an intensive use of graphical programming and thereby inherits characteristics contributing to execute quality control in complex applications. Our idea is also to always place the designer in a situation which promotes a quality approach all along the development process.

The first part of the paper gives a short description of the Lacatre design principles relying on four different axis -behavioural, transformational, abstraction level and application phase-.

The second part will be devoted to the presentation of the Lacatre methodology and the methodology assistant. This assistant takes advantage of the Lacatre environment specificity, allowing an incremental development and providing an assistance adapted to the current context of the design.

We will introduce short examples to illustrate the Lacatre design principles and methodology and the use of an assistant.

\section{DESIGN PRINCIPLES}

The use of graphical tools can be very convenient for the realisation of complex real-time systems, as it is in other domains, such as mechanical or electronic engineering (Hinchey, 1994). Graphical design and programming with Lacatre provide a precise and synthetic view of the various aspects of a multitasking real-time application and produce a look-and-feel documentation with a high level of legibility. That guided us all along the definition and design of the Lacatre environment, which essentially relies on a graphical design and programming language. Such a designed application gives a set of diagrams with a well defined syntax and semantic. These diagrams are made up with graphical objects -the Lacatre atomic objects, images of those involved in standard industry real-time executives- and graphical links -the Lacatre actions, the kernel system calls-. Associated to this diagram, the designer can, if needed, take advantage of the textual dual form (Figure 1), which completes the documentation of the application design: it highlights the very detailed algorithmic structures of the application.

A real-time application can be analysed throughout various views and we primarily extract four main ones:

- The behavioural axis gives an idea of the dynamic behaviour of an application from the multitasking and communication/synchronisation points of view.

- The transformational axis defines how and where is done the real-time application data processing -data flow modelling- (Petit, 1994). 


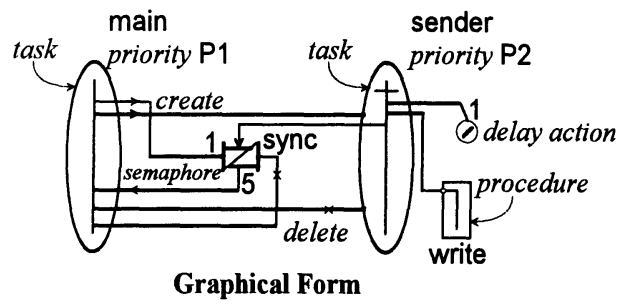

In fact, the texts in italic never appear on the graphical form

the textual form below explains the graphical notations

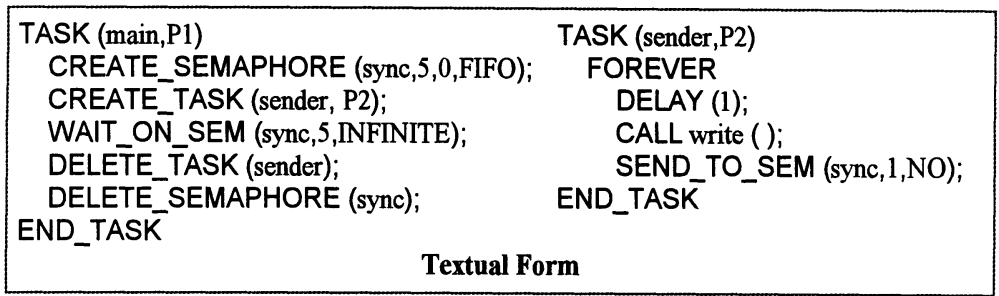

Figure 1 Diagram and textual form for a tasks synchronisation application.

- The abstraction level axis: the high level is closely linked with the application issues applicative objects- and the low level defines the interface with the target computer system Lacatre atomic objects-, executing the real-time application program. The applicative objects, used at the Preliminary Design step, are very general components able to express the main functionality of an application. The atomic objects, the designer handles at the Detailed Design step, are functionally very close to the target system possibilities: task, semaphore, interrupt, resource... Their use are straightaway dependant of the chosen target system (Schwarz and Skubich, 1993).

This abstraction level axis will be developed and illustrated later in the paper.

- The applicative phase axis: several phases may be distinguished within a real-time application execution which correspond to the various steps of the application execution and which are bound with its own time constraints. One can distinguish four major phases:

1.The initialisation phase which launches the application by defining all the various components of the application; this phase can be purely sequential and usually is not time critical but only time ordered.

2. The termination phase when it exists, is symmetric to the initialisation one -takes charge of the objects deletion- and may be purely sequential too (usually not time critical); it is associated to the program shutdown.

3. The kernel phase describes the normal running mode of the application; it corresponds to the dynamic behaviour of the real-time application in standard situations with respect of all its constraints and in particular the temporal ones.

4. The exception phase deals with the exception handling that are bound to the application side effects (dynamic behaviour in non standard circumstances); according to the application complexity, this phase can be integrated to the kernel or to the termination phase and is essential in real-time applications. 
The programmer separately designs these different phases, associating to each of them a design tracing with the appropriate objects and primitives. The general design document of the application is achieved by the superposition of all the basic tracings (Figure 1 and 2). Therefore, the applicative phase axis enhances the system simplification by partitioning: it is more efficient than a simple modularity -in fact, modularity can be added on each phase-

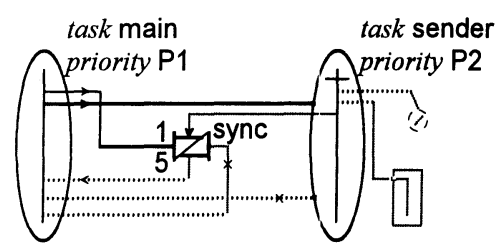

Initialisation phase

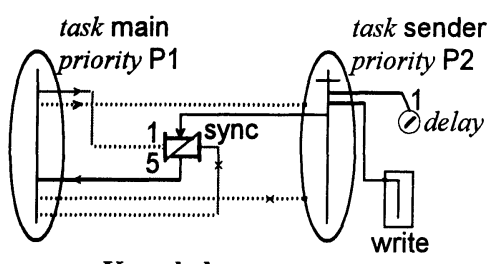

Kernel phase

Figure 2 Two phases of the previous real-time application.

The Lacatre atomic objects act as an overlayer upon most of the off-the-shelves real-time executives and thanks to a modelling of such an executive, it is possible to complete the design with the target code generation (currently $\mathrm{C}$ language for the iRMX kernel).

The design quality of real-time applications is favoured by a strict adequation between the abstraction level and the object resolution: on the one hand, the higher the abstraction level is (Preliminary Design), the simpler the graphical symbolism and the connection rules are and, on the other hand, at a low abstraction level (Detailed Design), the symbolism is more detailed and the connection rules are more elaborated justifying thus to a great extent the automatic assistance given by the tool. We note that the introduction of the high level objects in the environment is a clear incitement to maintainability improvement, at a reasonable cost and promotes a coherent approach to complex systems.

This design formalism is not necessarily adapted for correctness verification which requires usually formal methods. Therefore when necessary, an automatic translation into an other formalism better suited for formal methods like for example Communicating Real-time State Machines (Shaw, 1992), can be done. This solution (CRSM) is carried out in the Lacatre environment but other translations may also be possible e.g. Petri nets.

\section{LACATRE METHODOLOGY}

\subsection{Aim}

The quality control, in CIM applications as well as in embedded systems, requires a global approach and becomes essential as soon as dependability is tackled (reliability, availability, maintainability, safety...). The development of a reliable system is based on the combined use of a set of methods (Laprie, 1989; Laprie, 1993; Laprie, 1995):

- two classes contributing to achieve quality that is, to avoid during the design and the programming, the emergency and introduction of faults (fault prevention methods) and to provide, for example thanks to redundancy, a service consistent with the specification in spite of faults (fault tolerance methods); 
- and two others for quality validation that is, to reduce the occurrence (number and severity) of faults (fault elimination methods like testing) and to anticipate the faults presence (prediction methods like quantitative approaches).

The process described in this paper deals with the methods for avoiding faults (methodology approach) and with the prediction methods (quantitative measurements). It covers both the preliminary design steps and the detailed design steps with a view to an automatic target code generation. This process relies on a phases and steps chain whose aim is to minimise omissions, to avoid inconsistency, to favour reusability (atomic objects and applicative objects) and to master complexity thanks to a design based on drawings.

As the use of CASE tools with a too strong rigidity in the design process is often at the beginning of a rejection on behalf of the users (Gibson and Snyder, 1991), we have chosen a small steps chain with flexibility and adaptability. Thus, the methodology assistant has to advise, contextually and in a clever manner, the designer to incite him to reach a good design and development, and to assist him in the selection and construction of a final solution satisfying the quality constraints. During the following description of the design principles, all illustrations will be taken out from the same example: a simple monitoring of an industrial transfer system.

\subsection{Description}

During the preliminary design steps, the programmer defines a possible application decomposition in terms of applicative objects and theirs relations. Each object will be defined by:

- a name, image from the real world or the design step (inherited from the specification stage);

- a description of the offered services (inherited from the specification stage);

- architectural features (for instance, the applicative object is distributed or not...);

- an interface.

The initial steps includes four stages each of which is made up with operations and activities to do, with useful information for each activity, with control on the activities results, with the appropriated documentation to produce.

\section{Context analysis}

The designs starts with the enumeration of the inputs and outputs of the system. They define the connections or interface with the monitored or controlled world (Figure 3 (a)).

\section{Architecture definition}

It consists in a general architecture description of the real-time application in terms of Lacatre applicative objects (e.g. services required or provided by these objects) (Figure 3 (b)).

Definition, phase by phase, of all the relations between the applicative objects:

This step is a basic refinement of the previous one and is focusing on the communication channel between the objects (Figure 4). 


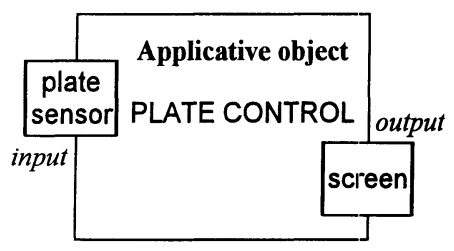

(a) Title diagram of the application
PLATE CONTROL

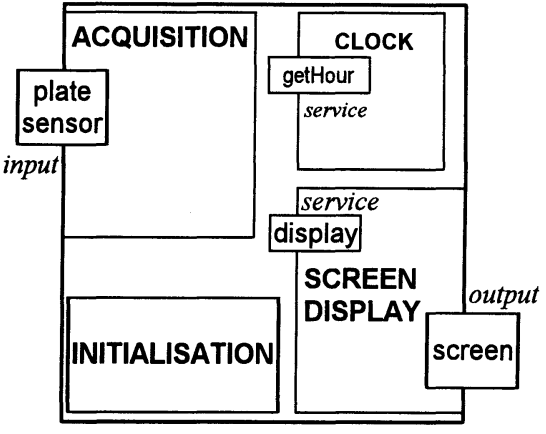

(b) General architecture of the application

Figure 3 Applicative objects of the monitoring part of a transfer system.

PLATE CONTROL

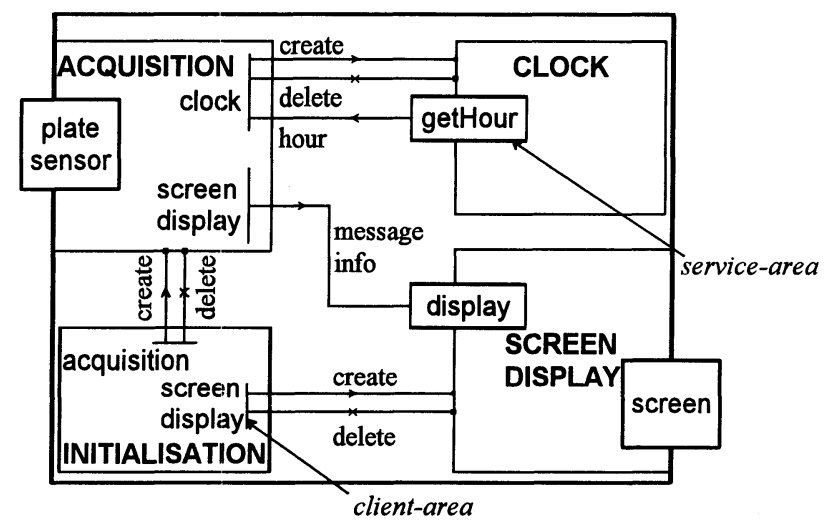

Figure 4 Definition of the communications.

It is decomposed into:

- the definition of all the relations: control flow (create, delete, suspend...) and data flow (message passing...);

- the introduction, if necessary, of connection areas (client-area, service-area in Figure 4 and import / export operators in Figure 5 and 6) in the applicative objects in order to provide anchor points for the communication channels.

\section{Choice of the communication channel}

The purpose of this step is to refine the communication between the applicative objects. The different communication possibilities the designer can use, are then proposed -or even imposed- indicating clearly the implications with regard to efficiency, reusability, embedding, 
distribution on a multiprocessor architecture... This choice has obviously to be guided by quality constraints. There are mainly two communication possibilities:

- Access to an applicative object through a single entry point identified by a simple procedure call. Thus, the only thing to do is to make available to the outside of the application object the name of that procedure (entry point InitClock).

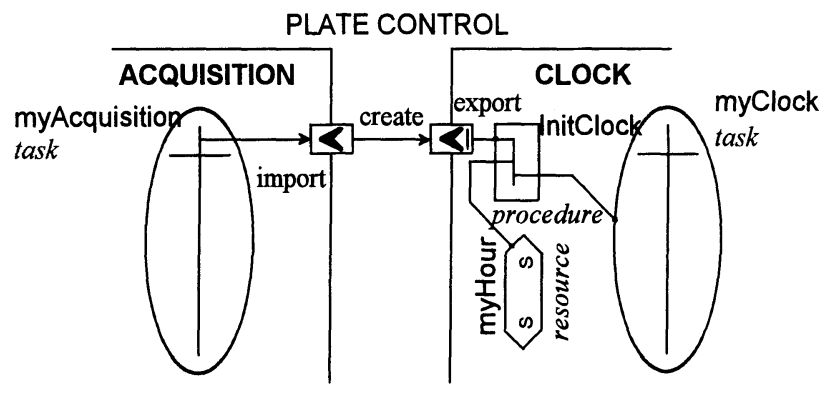

Figure 5 Communication channel through an import / export procedure entry point.

- Data flow through direct access to the atomic objects involved in the construction of the applicative objects, otherwise said, a partial inside view of the applicative object is accessible. From a dynamic point of view, the designer has a better control of the behavioural aspect of his application and thus, of the time essential in real-time applications.

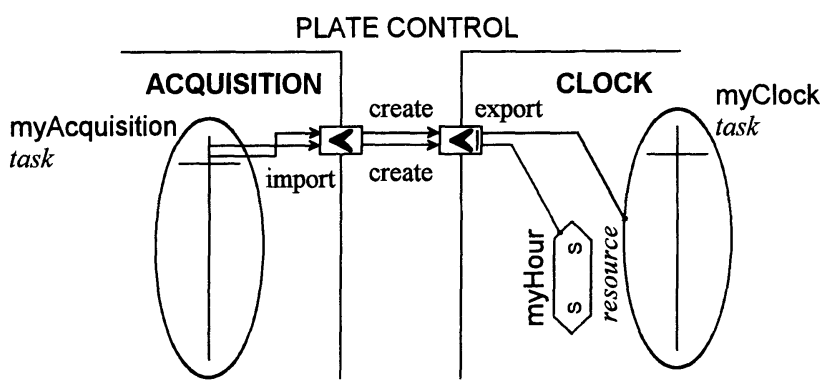

Figure 6 Communication protocol through a direct access to the atomic objects.

At this stage of the development, the application architecture is well defined. A synthetic check up can be proposed and is essentially based on:

- the list of objects to develop, with their specification and some indications about a possible reuse of already existing applicative objects (search, by name or keywords, in the object data base of applicative objects) (Aubry, 1995);

- the different views of the developed application (hierarchical, synthetic...). 
Thus, during the detailed design steps, all the previous defined applicative objects, issued from the preliminary design steps, have to be designed in detail with the use of the Lacatre atomic objects and actions. The proposed method follows two specific axis:

- definition of the services between applicative objects, if necessary;

- definition or end of the definition of the applicative object itself.

The intensive use of graphical design in this step reduces the programmer's work, especially by relieving him of technical and low level detail -necessarily textual-. During this activity, he is guided by the application phase axis (kernel phase, initialisation and termination phases and possibly exception phase) (Figure 2) and by the graphical tool controls which avoid any redundancy and connection mistakes.

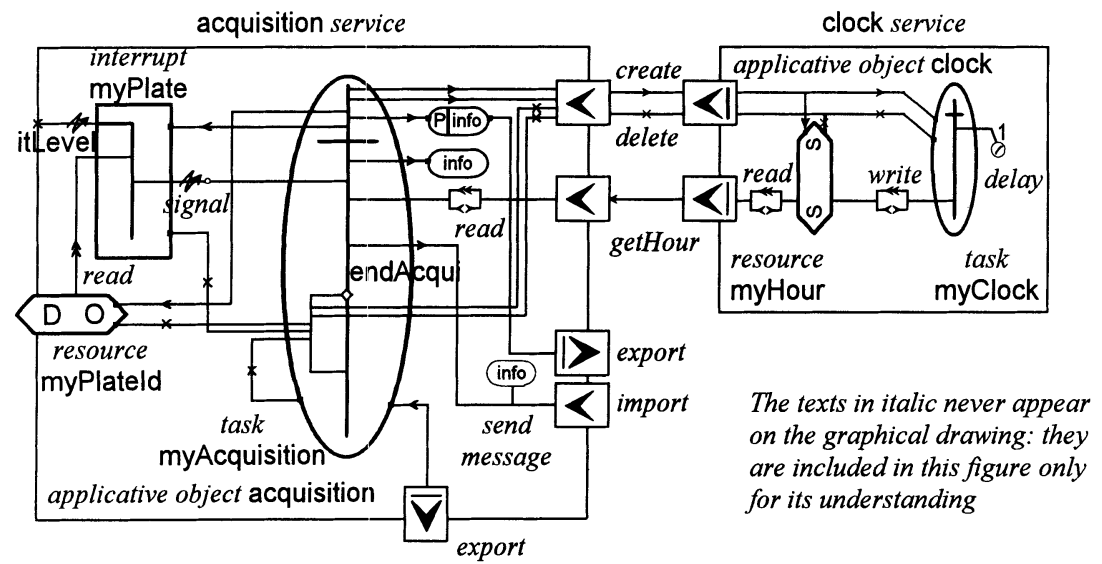

Figure 7 Applicative object acquisition and clock (detailed view).

After this step, all the various components of the application are completely defined and an automatic target code generation can take place.

Allowing incremental developments, this methodology can be well suited to very large systems construction in which the applicative objects may be designed and implemented by different groups of people. Indeed, at a given time, they may not all be at the same level of progress and a precise evaluation of each level of progress becomes absolutely necessary (step in the development methodology).

\section{METHODOLOGY ASSISTANT}

\subsection{Main features}

The Lacatre methodology, presented in the former section, applies both the Preliminary Design (software architecture) and the Detailed Design. This methodology is supported by a methodological assistant (Burlon and Tagliati, 1990). Its purpose is to help, all along the 
development process, the designer by providing contextual methodological advice and particular guidelines for a good respect of the methodology. It becomes one of the principle communication interfaces between the Lacatre environment and the designer.

The Lacatre methodology assistant will provide the designer with a trace of the development process he adopted for a given design. He may analyse it and/or have it in mind for the next equivalent project.

This tool provides several kinds of classical functionality (illustrated in the next section) (Burlon and Tagliati, 1990):

\section{Guidance through the method steps:}

Thanks to this guidance, the designer is able to carry out the methodology process himself (phases and steps with the associated activities and operations for each of them). It completely defines the guideline through the development method.

\section{Methodological advice:}

Step by step, the assistant supplies the designer with contextual advice and with information about goals and criteria to observe during the development.

\section{Information and reporting about the design:}

A general view of the current design state is provided.

\section{Check facilities:}

The help of the methodology assistant is also based on a set of checks to be performed in order to detect design faults.

\section{Quality assistance:}

The assistant has also to ensure a better monitoring of the quality of the developed application (static measurement approach on the product) (Fenton, 1991). The specific attributes, defined in our assistant are the maintainability, the reliability, the reusability and the portability.

\subsection{Illustration of the assistant}

Some special features of the assistant will be illustrated using our transfer system.

\section{Methodological advice}

Among the constraints required by an application, the encapsulation aspect can be an important one. In such a case, the assistant will rather recommend, during the choice of a communication protocol (applicative object CLOCK, step 4. in the preliminary design), a procedure call (InitClock) than a direct access to a set of Lacatre atomic objects. The tool can produce as an output, the following control panel in answer to such a methodological advice.

Table 1 A methodological advice

\begin{tabular}{|c|c|c|c|c|}
\hline Phase & Step & Activity & $\begin{array}{l}\text { Non functional } \\
\text { requirement }\end{array}$ & Assistant advice \\
\hline $\begin{array}{l}\text { preliminary } \\
\text { design }\end{array}$ & step $n^{\circ} 4$ & $\begin{array}{l}\text { Communication } \\
\text { channel choice }\end{array}$ & embedding & $\begin{array}{l}\text { access to an } \\
\text { applicative object } \\
\text { through a single entry } \\
\text { point } \\
\text { (procedure call) }\end{array}$ \\
\hline
\end{tabular}




\section{Information and reporting about actual design}

At each stage of the development, the designer can obtain a general view concerning the design states of his applicative objects. In the example, the state diagram Figure 8 may be displayed for the applicative object CLOCK when the user is in the step $n^{\circ} 4$-Choice of the communication channel- (Figure 5).

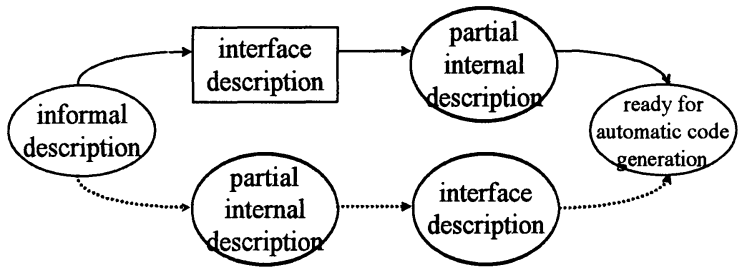

Figure 8 Simplified development states for an applicative object.

This diagram shows several possible paths to build an applicative object and illustrates the flexibility aspect of the method. The ideal way to go from the informal description to the final applicative object construction is given by the continuous line. An alternate way is possible via the doted line. The state, surrounded by a rectangle, indicates the current design state of the applicative object. Thus, the designer evaluates the necessary work to obtain a ready for automatic code generation applicative object. This gap is highlighted by the difference between Figure 5 and Figure 7 which completely defines the applicative object CLOCK. The diagram Figure 8 is a very simplified one. The complete drawing integrates the different development states corresponding to each axis of the Lacatre design principles and especially to the application phase axis (kernel phase, initialisation and termination phases and possibly exception phase). Thus, the different nodes are split.

\section{Check facilities}

The help is based on a set of coherence checks to detect design faults. For example:

- loop detection in the relationship between applicative objects;

- harmful use of some primitives in special situations for some specific real-time operating systems (e.g. during interrupt servicing);

- static validation of the correct use of the atomic objects (correct sequence of the used primitive calls: create, use, delete)...

\section{Quality assistance}

The quality assistance is based on a quality tree derived from Mc Call's and Bowen's model (with a three level hierarchy of factors, criteria and metrics) (Bowen, 1985; Kitchenham, 1992).

The main preoccupation is to evaluate the design quality, according to different possible views (application, applicative objects, programmable objects) and thanks to a set of easily and classical measurable indicators (Fenton, 1991) (Mc Cabe's cyclomatic number (Mc Cabe and Butter, 1989)) but also some new and completely specific ones inherited from the hierarchical relative correctness verification (Szmuc, 1991; Szmuc, 1992; Aubry, 1995).

The Lacatre quality assistant deals with four usual factors used to estimate the design quality of complex systems (Figure 9). 


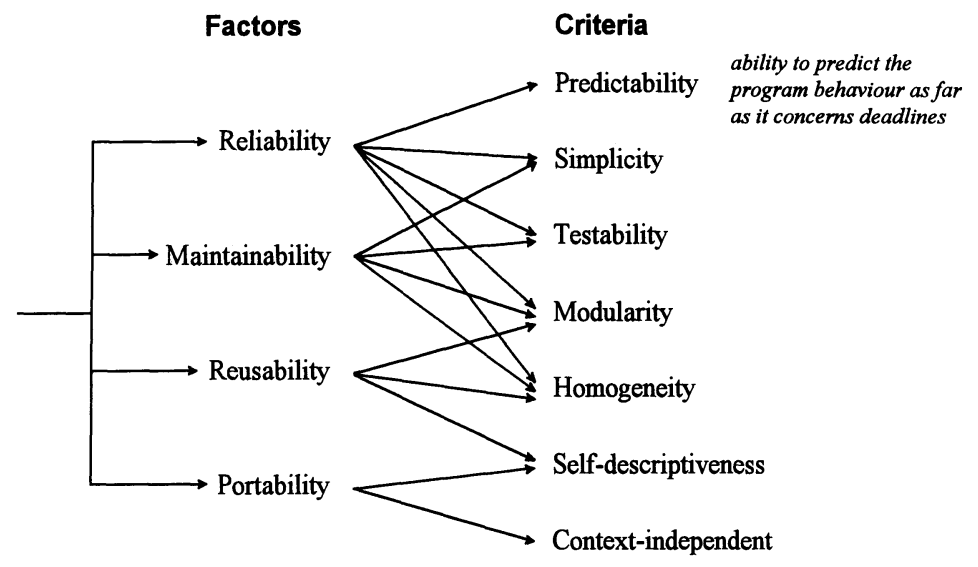

Figure 9 Lacatre quality tree (factors, criteria).

In accordance with the current progress state of the project, the quality assistant guides the user to reach, first of all, good maintainability and reliability features. The achievement of this goal is obtained by controlling the complexity of, firstly, the applicative objects and then, the complexity of the programmable atomic objects of the detailed design. For example, the applicative object simplicity can be checked with respect to thresholds set up by the project manager (Figure 10).

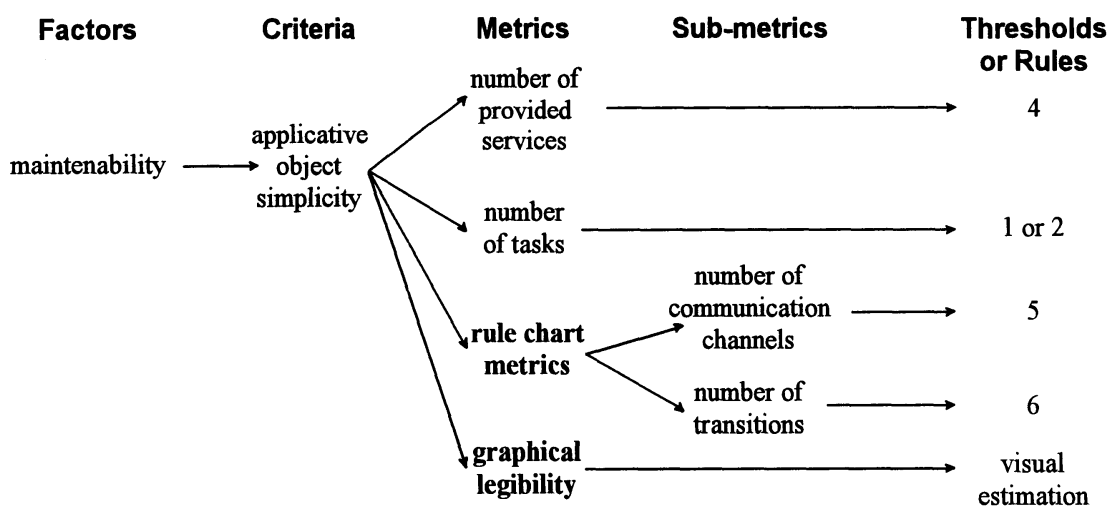

Figure 10 Maintainability factor.

The graphical legibility metric can be estimated qualitatively by static verification technics which are checking the respect of user friendly rules.

The rule chart metrics are derived from characteristics (such as communicating channels properties, transitions) defined during the correctness verification steps (Szmuc, 1992) thanks to a Communicating Real-time State Machines modelling (Shaw, 1992) of the Lacatre design. 


\section{CONCLUSION}

The interest of a methodology assistant, which is under definition, is to contribute to the design of better quality real-time systems. It allows, step by step, to accompany the developer by giving him a view on the current design state. The use of such an assistant in conjunction with a graphical design environment and with correctness verification possibilities (Szmuc, 1991; Szmuc, 1992) contributes to reach a better quality control in the real-time applications development and to satisfy non functional requirements such as dependability. Moreover, a quality assistant is grafted to this environment to perform a static evaluation of the design quality from a Lacatre quality tree.

\section{REFERENCES}

Aubry, R., Maranzana, M. and Schwarz, J.J. (1995) Quality assistance for a graphical development of real-time applications. 4th Software Quality Conference, Dundee, Scotland.

Bowen, T.P. (1985) Specification of software quality attributes. New York: Rome Air Development Centre (RADC), 3, Technical report RADC - TR-85-37.

Burlon, R. and Tagliati, M. (1990) APSIS: Knowledge based assistance for system development. Technique et Science Informatiques, AFCET Bordas, 9(2), 176-182.

Fenton, N.F. (1991) Software metrics: a rigorous approach. chapman\&hall, London.

Gibson, M.L. and Snyder, C.A. (1991) Computer aided software engineering: facilitating the path for true software and knowledge engineering. International Journal of Software Engineering and Knowledge Engineering, 1(1), 99-114.

Hinchey, M.G. (1994) Visual Methods in Real-Time Programming. 19th IFAC/IFIP Workshop on Real-Time Programming, Reichenau, Germany, June 1994.

Kitchenham, B. (1992) Development in software metrics in the last decade. Eurometrics' 92 , European conference on quantitative evaluation of software and systems, practical and theoretical aspects, Brussels, Belgium, April 1992, 17-26.

Laprie, J.C. (1989) Hardware and software dependability evaluation. 11th International IFIP Workshop, 28 august-1 september, San Francisco, USA, 109-114.

Laprie, J.C. (1993) Dependability: from concepts to limits. Symposium on Safety of Computer Control System (SAFECOMP'93), Poznan, Pologne, 157-168.

Laprie, J.C. (1995) Guide de la sûreté de fonctionnement. Laboratoire d'Ingénierie de la Sûreté de fonctionnement (LIS), Cépadues Edition, Paris.

McCabe, T.J. and Butter, C.W. (1989) Design complexity measurement and testing. Communication of the ACM, 32(12), 1415-1425.

Petit, J.F., Schwarz, J.J., Maranzana, M. and Skubich, J.J. (1994) Data flow Modelling in Real-Time Multitasking Graphical Design. IEEE Workshop on RTA, Washington, USA.

Schwarz, J.J. and Skubich, J.J. (1993) Graphical Programming for Real-Time Systems. Control Engineering Practice - IFAC, 1(1), Pergamon Press.

Shaw, A.C. (1992) Communicating Real-Time State Machines. IEEE Transaction on Software Engineering, 18(9), 805-816.

Szmuc, T. (1991) Partial and total relative correctness for analysis of concurrent systems, Journal of Applied Mathematics and Computer Sciences, 1(1), 27-34.

Szmuc, T. (1992) Relative correctness for analysis of real-time programs, IFAC/IFIP Workshop on Real-Time Programming 1992, Pergamon Press, 173-177. 


\section{BIOGRAPHY}

Regis Aubry is a senior lecturer of Computer Science at Institut National des Sciences Appliquées (INSA) de Lyon. His research interests include software engineering, complex system and software quality, project management and groupware. He is responsible for software engineering, quality assurance and metrology courses.

Regis Aubry is a graduate of the Computer Sciences Department at INSA and he received a doctorate in operating system. (e-mail: Regis.Aubry@if.insa-lyon.fr).

Mathieu Maranzana is a senior lecturer of Computer Science at Institut National des Sciences Appliquées (INSA) de Lyon. His research interests include compiler construction, software design, software engineering and graphical design tools and environments.

Mathieu Maranzana is a graduate of the Computer Science Department at INSA and he received a doctorate in compiler construction (e-mail: Mathieu.Maranzana@if.insa-lyon.fr).

Jean-Jacques Schwarz is a assistant professor of Computer Science at Institut National des Sciences Appliquées (INSA) de Lyon, head of the Laboratoire d'Ingénierie de l'Informatique Industrielle (L3i). His research interests are graphical design and software architectures for real-time applications, enginering and reengineering of complex computer systems.

Jean-Jacques Schwarz is a graduate of the Electronics Department at INSA and he received a doctorate in time series analysis. He is a member of the IEEE Computer Society (e-mail: Jean-Jacques.Schwarz@if.insa-lyon.fr). 